\title{
Visualization of Plaque/Vessel Interfaces using FIB-SEM
}

\author{
George Wetzel, ${ }^{1}$ Susan M. Lessner ${ }^{2}$ \\ 1. Clemson University Electron Microscopy Facility, Anderson, SC \\ 2. University of South Carolina School of Medicine, Department of Cell Biology \& Anatomy, \\ Columbia, SC
}

Delamination or tearing between tissue layers is a material failure mode observed in several clinically significant cardiovascular pathologies, particularly in arterial dissection. Delamination also occurs as a result of iatrogenic damage to vascular walls, as in the example of intimal flap formation during balloon angioplasty. ${ }^{1}$ To better understand vascular tissue delamination failure from a mechanical perspective, we have performed experimental plaque delamination studies in mouse models of atherosclerosis, ${ }^{2,3}$ which show that failure typically occurs at the luminal surface of the internal elastic lamina (IEL), an elastic membrane which separates the intimal and medial tissue layers in the aorta. The microstructural mechanism of failure at this interface has yet to be identified, but fibrous elements of the extracellular matrix, particularly collagen fibers, are believed to contribute to the observed adhesive strength. ${ }^{2}$ Characterizing the ultrastructural organization of matrix fibers at the plaque-IEL interface will provide critical information needed to define the interface geometry for computational simulations of tearing failure. Here we examine the use of FIB-SEM as a potential tool to help characterize the 3-dimensional ultrastructure of the plaque-IEL boundary.

\section{Methods}

Mice genetically deficient in apolipoprotein E (apoE KO) were used as an animal model of atherosclerosis. After being maintained on a high-fat diet for 12 months to induce advanced atherosclerotic plaque development throughout the descending aorta, the mice were euthanized by an overdose of anesthetic and the aortas were perfusion fixed with dilute Karnovsky's fixative at mean arterial pressure. Tissue samples were processed and stained using a modification of the OTO (osmium-thiocarbohydrazide-osmium) method, dehydrated in graded alcohols, and embedded in Durcupan ACM resin for electron microscopy.

Fixed and embedded samples were sputter coated with approximately $10 \mathrm{~nm}$ of platinum to form a conductive layer prior to ion beam milling. Samples were loaded into a Hitachi NB5000 nanoDUE'T Focused Ion Beam and Scanning Electron Microscope (FIB-SEM) system for milling and subsequent image capture. Using the Mill\&Monitor $\bigcirc$ feature, serial slices were removed from the sample block using the ion beam, with an electron micrograph acquired after each slice. These images were stacked together to form a three-dimensional model.

The specific region of interest was the interface between the vessel wall and plaque. After the region of interest had been located and oriented such that the interface would be visible to the SEM after sputtering, a box trench was cut into the sample using a high current, $40 \mathrm{kV} \mathrm{Ga}+$ beam. A tungsten cap was deposited to protect the region of interest, and the cross section face further polished with a lower energy ion beam to remove any artifacts. Serial slices spaced $18 \mathrm{~nm}$ apart were removed from this face using a $0.07 \mathrm{nA}, 40 \mathrm{kV}$ ion beam. 396 iterations were done for a total sampling thickness of $7 \mathrm{um}$. Milling time for each slice was set at $1 \mathrm{~min}$. Images of the sliced face were acquired at a magnification of $7 \mathrm{kx}$ using a $3 \mathrm{kV}$, high probe current beam to minimize imaging depth and increase backscattered electron yield. Images were acquired 
using the ExB in-column backscattered electron detector with a capture time of 160 s for an increased signal to noise ratio.

All images acquired using Mill\&Monitor(C) were loaded into Avizo Ver.8 for 3D rendering. Orthoslice projections were viewed along the XY, ZY, and ZX planes.

\section{Conclusions}

FIB-SEM provides a potentially useful tool to uncover 3-dimensional ultrastructural organization of mouse aortic wall at the plaque-IEL interface. However, due to thermal drift, spatial resolution is best in the plane orthogonal to the direction of ion milling. Future work will focus on image analysis and 3-D reconstruction of the interface geometry.

\section{$\underline{\text { References }}$}

1. Honye, J, Mahon DJ, Jain A, White CJ, Ramee SR, Wallis JB, al-Zarka A, Tobis JM, Circulation, 85(3), 1012-25 (1992).

2. Wang Y, Johnson JA, Fulp A, Sutton MA, Lessner SM, J. Biomech., 46, 716-722 (2013).

3. Wang Y, Ning J, Johnson JA, Sutton MA, Lessner SM, J. Biomech., 44(13), 2439-2445 (2011).

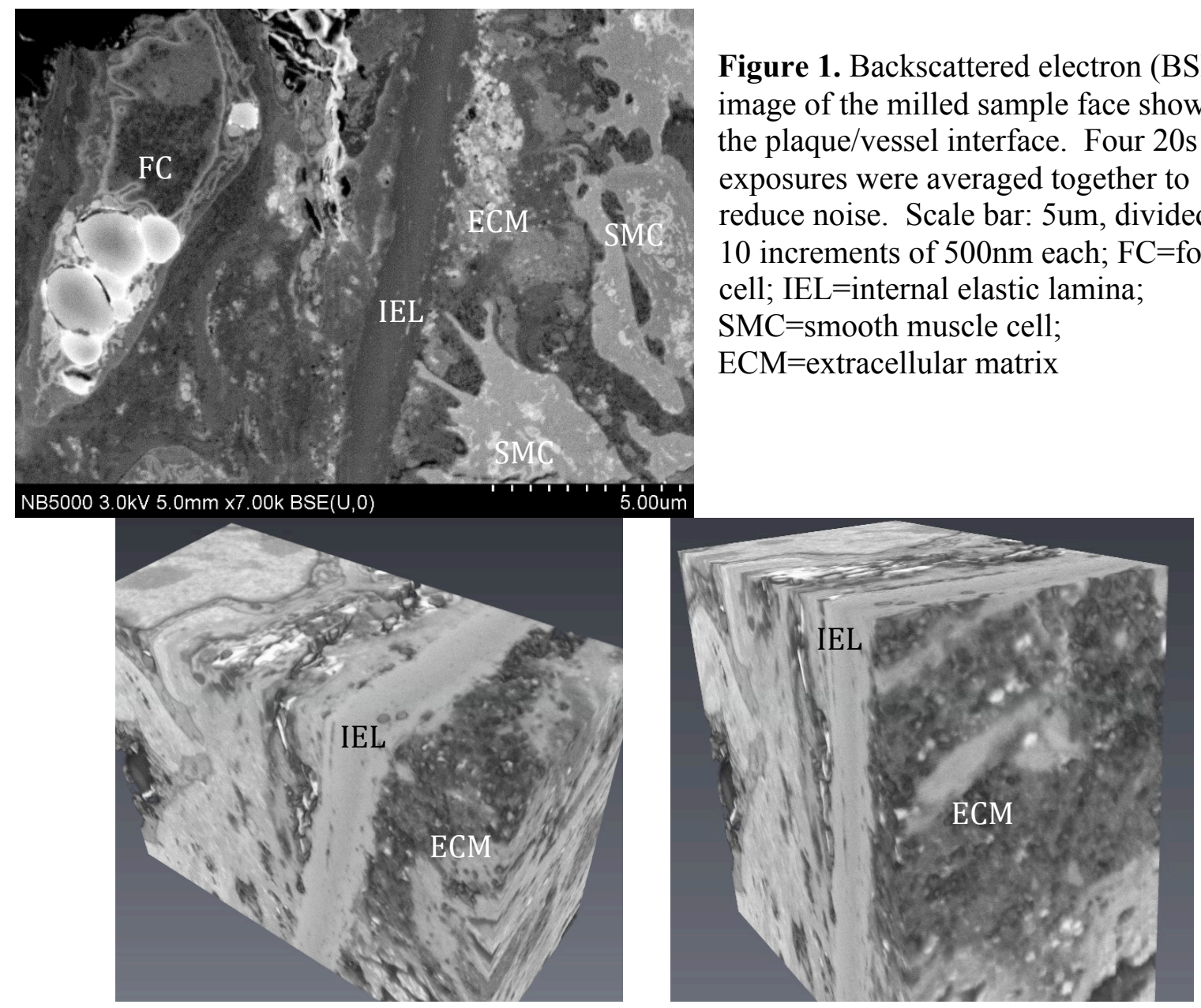

Figure 2. 3D stack printed in reverse contrast to resemble TEM contrast. Model has been cropped to reveal details in the ECM adjacent to the IEL. 